\title{
$\gamma \delta$ T cells: an immunotherapeutic approach for HIV cure strategies
}

\author{
Carolina Garrido, ${ }^{1,2}$ Matthew L. Clohosey, ${ }^{1,2}$ Chloe P. Whitworth, ${ }^{1,2}$ Michael Hudgens, ${ }^{3}$ \\ David M. Margolis, ${ }^{1,2,4,5}$ and Natalia Soriano-Sarabia ${ }^{1,2}$ \\ ${ }^{1}$ UNC-HIV Cure Center, ${ }^{2}$ Departments of Medicine, ${ }^{3}$ Biostatistics, ${ }^{4}$ Microbiology and Immunology, and \\ ${ }^{5}$ Epidemiology, University of North Carolina at Chapel Hill, Chapel Hill, North Carolina, USA.
}

\begin{abstract}
Current strategies aimed to cure HIV infection are based on combined efforts to reactivate the virus from latency and improve immune effector cell function to clear infected cells. These strategies are primarily focused on $C D 8^{+} T$ cells and approaches are challenging due to insufficient HIV antigen production from infected cells and poor HIV-specific $C D 8^{+} T$ cells. $\gamma \delta$ T cells represent a unique subset of effector $T$ cells that can traffic to tissues, and selectively target cancer or virally infected cells without requiring MHC presentation. We analyzed whether $\gamma \delta$ T cells represent a complementary/alternative immunotherapeutic approach towards HIV cure strategies. $\gamma \delta \mathrm{T}$ cells from HIV-infected virologically suppressed donors were expanded with bisphosphonate pamidronate (PAM) and cells were used in autologous cellular systems ex vivo. These cells (a) are potent cytotoxic effectors able to efficiently inhibit HIV replication ex vivo, (b) degranulate in the presence of autologous infected $\mathrm{CD4}^{+} \mathrm{T}$ cells, and (c) specifically clear latently infected cells after latency reversal with vorinostat. This is the first proof of concept to our knowledge showing that $\gamma \delta$ T cells target and clear autologous HIV reservoirs upon latency reversal. Our results open potentially new insights into the immunotherapeutic use of $\gamma \delta$ T cells for current interventions in HIV eradication strategies.
\end{abstract}

Conflict of interest: DMM holds public stock in Gilead Sciences, outside of the content of this work.

Submitted: January 24, 2018

Accepted: May 10, 2018

Published: June 21, 2018

Reference information: JCI Insight. 2018;3(12):e120121. https://doi.org/10.1172/jici. insight.120121.

\section{Introduction}

The latent human immunodeficiency virus (HIV) reservoir within resting memory $\mathrm{CD}^{+}{ }^{+} \mathrm{T}$ cells is the major barrier to efforts to eradicate persistent infection (1-4). Therapeutic approaches that allow the clearance of latent replication-competent HIV are thus needed. Current strategies are based on using latency reversal agents (LRAs) to induce viral antigen expression $(5,6)$ to allow immunological clearance. This clearance part has been mainly based on $\mathrm{CD}^{+} \mathrm{T}$ cells $(7,8)$. However, $\mathrm{CD} 8$-based therapies can be challenging due to insufficient HIV antigen expression on latently infected cells, diminished function of specific $\mathrm{CD} 8^{+} \mathrm{T}$ cells $(9,10)$, and escaped HIV variants (7). Given their unique characteristics, we hypothesized that $\gamma \delta \mathrm{T}$ cells may constitute an attractive alternative or complementary strategy to current approaches to cure HIV infection.

One of these distinct characteristics relates to antigen recognition. In contrast to the majority of $\alpha \beta \mathrm{T}$ cells that recognize antigen peptides bound to major histocompatibility complex (MHC) class I or II, $\gamma \delta \mathrm{T}$ cells, specifically the most prevalent peripheral subset, V 22 cells, mainly recognize nonpeptidic phosphorylated metabolites of isoprenoid biosynthesis (11). These metabolites include (E)-4-hydroxy-3-methyl-but-2-enyl pyrophosphate (HMBPP) $(12,13)$ and isopentenyl pyrophosphate (IPP) (14), and their recognition does not require conventional $\mathrm{MHC}$-antigen presentation $(14,15)$. Of interest, aminobisphosphonates (N-BPs) such as pamidronate (PAM) and zoledronate $(\mathrm{Zol})$ are drugs that modulate isoprenoid metabolism, indirectly augmenting IPP production and activating V $\delta 2$ cells (16). Once activated, V $\delta 2$ cells are potent cytotoxic effectors (17) against malignancies $(18,19)$ and HIV-infected cells (20-23). We and others have previously demonstrated that $\mathrm{V} \delta 2 \mathrm{~T}$ cells can inhibit active viral replication $(17,24)$. In addition, expanded V $\delta 2 \mathrm{~T}$ cell immune adjuvant functions include induction of dendritic cell maturation and activation of HIV-specific $\mathrm{T}$ cell responses (25), which are of special interest as a future complementary use of $\gamma \delta \mathrm{T}$ cells in adoptive cell transfer strategies. In this regard, a small clinical trial with antiretroviral therapy-naive (ART-naive) HIVinfected donors reported expansion of V $\delta 2$ cells, dendritic cell activation, and increased HIV-specific T cell responses after in vivo administration of Zol and IL-2 (26). Altogether, these studies suggest that $\gamma \delta \mathrm{T}$ cells 
A

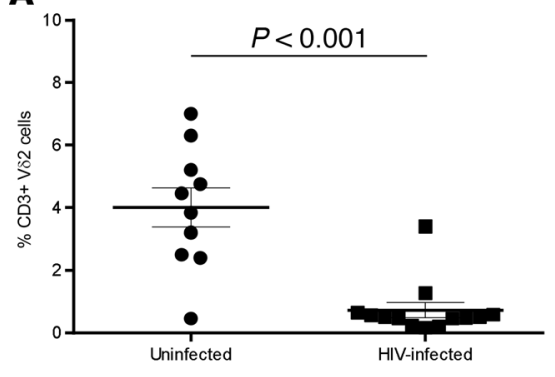

B

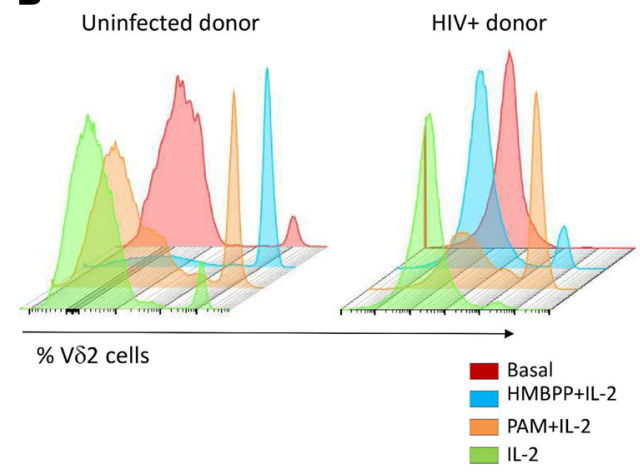

C

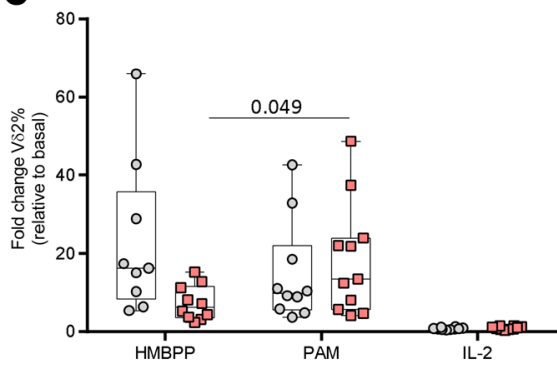

Figure 1. Expansion of $\mathbf{V} \boldsymbol{\delta} \mathbf{2}$ cells after $\mathbf{6}$ days of culture. (A) Greater V $\delta 2$ cell frequency in uninfected donors. PBMCs of uninfected ( $n=10$ ) and HIV-infected donors $(n=13$ ) were stained for CD3 and V $\delta 2$ and analyzed by flow cytometry. As expected, uninfected individuals showed a statistically higher percentage of $V \delta 2$ cells compared with HIV-infected donors. Data represent mean \pm SEM (Mann-Whitney $U$ test, $P<0.001$ ). (B) Representative histograms showing V $\delta 2$ cell expansion. PBMCs from uninfected (left histogram) or ART-suppressed HIV-infected donors (right panel) were incubated for 6 days using HMBPP + IL-2 (blue), pamidronate (PAM) + IL-2 (orange), or IL-2 alone (green). (C) V $\delta 2$ cells from HIV-infected individuals expand in response to PAM and IL-2. V 22 cell fold change relative to basal cell numbers is represented. HIV-infected donors' response to HMBPP was lower, not statistically significant after FDR adjustment, compared with uninfected individuals (FDR $P=0.11$ ). Response to PAM and IL-2 was similar between uninfected and HIV-infected donors (FDR $P=0.29$ ). Response to HMBPP and PAM in uninfected donors was comparable (FDR $P=0.22$ ), while response to HMBPP in HIV-infected donors was statistically lower (FDR $P=0.04)$. Uninfected donors $(n=9)$ are represented with gray circles and HIV-infected donors $(n=11)$ with pink squares. Uninfected and HIV-infected donors were compared using Mann-Whitney $U$ test. HMBPP, PAM, and IL-2 conditions in uninfected donors and in HIV-infected donors were compared using Wilcoxon's signed-rank test.

deserve further investigation to assess their potential in interventions aimed to eradicate HIV infection taking advantage of the knowledge generated from the cancer field (27-29).

In the present study, we demonstrate $\gamma \delta \mathrm{T}$ cell targeting and elimination of reactivated HIV-infected resting $\mathrm{CD}^{+} \mathrm{T}$ cells ex vivo. To our knowledge, our work constitutes the first study that comprehensively analyzes the potential of $\gamma \delta$ T cells to clear persistent HIV infection in ART-treated, aviremic HIV-infected donors. We have performed ex vivo studies demonstrating cytotoxic and antiviral capacities of $\gamma \delta \mathrm{T}$ cells to specifically target and inhibit viral replication. Further, using autologous systems we demonstrate the specific clearance of latently HIV-infected cells by $\delta \mathrm{T}$ cells after latency reversal. This study provides rationale to further analyze the immunotherapeutic use of $\gamma \delta \mathrm{T}$ cells in HIV cure strategies.

\section{Results}

Comparison of VS2 cell expansion in HIV-infected and uninfected individuals. We compared different ex vivo experimental conditions to expand V $\delta 2$ cells from ART-suppressed HIV-infected donors $(n=13)$ and uninfected donors $(n=10)$. In this first approach we expanded the cells for 6 days and conditions included (a) HMBPP and IL-2, (b) PAM and IL-2, or (c) IL-2 alone. Basal V 22 cell percentages within CD3 ${ }^{+}$cells were analyzed by flow cytometry showing wide interindividual differences in uninfected individuals, and expected (30) profound depletion in HIV-infected donors (mean 4.0\% vs. 0.7\%, respectively; Figure 1A).

In uninfected individuals, HMBPP was a more potent inducer of V $\delta 2$ cell expansion compared with PAM, while cells from HIV-infected donors expanded better after PAM treatment (Figure 1, B and C, and Supplemental Figure 1A; supplemental material available online with this article; https://doi.org/10.1172/ jci.insight.120121DS1). Importantly, the fold change in expansion induced by PAM in HIV-infected donors was comparable to uninfected donors (Figure 1, B and C). In HIV-infected donors, after 6 days of culture, a mean of $5.3 \%$ of the total $\mathrm{CD}^{+}$cells present in the culture were V $\delta 2$ cells after HMBPP treatment, compared with $11.0 \%$ after PAM treatment (Supplemental Figure 1B). In summary, PAM allows efficient V $\delta 2$ cell expansion in HIV-infected donors.

V\&2 T cells from ART-treated HIV-infected individuals successfully expand after exposure to PAM. Based on these results, in further experiments peripheral blood mononuclear cells (PBMCs) were exposed to $25 \mu \mathrm{g} / \mathrm{ml}$ PAM and $100 \mathrm{U} / \mathrm{ml} \mathrm{IL}-2$ for a total of 14 days, to increase V 22 cell numbers. We expanded V 22 cells from 21 ARTsuppressed HIV-infected donors, to be used for different functional assays. Expansions were performed in the presence of antiretrovirals to avoid spread of infection. The average V $\delta 2$ cell expansion rate was $11.9 \%$ 
A

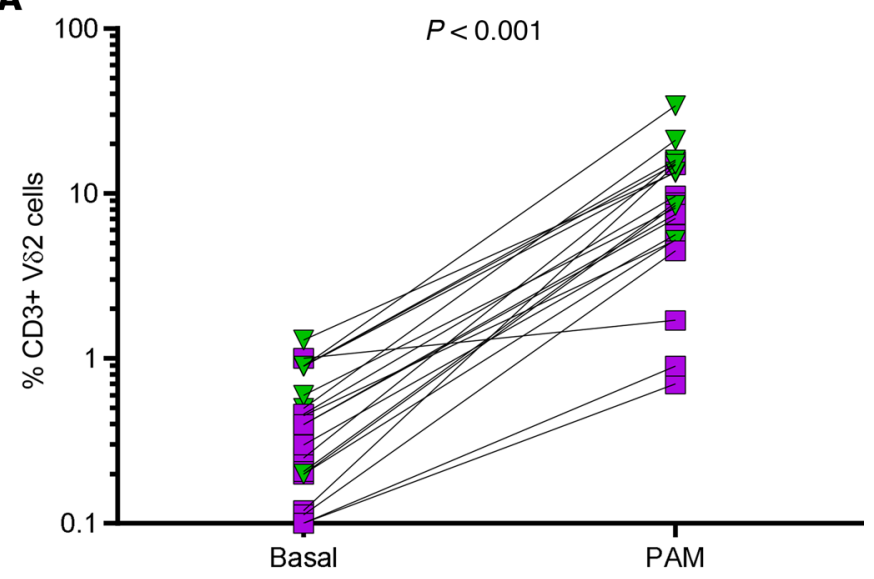

Figure 2. Expansion of V $\delta \mathbf{2}$ cells from ART-suppressed HIV-infected donors in response to pamidronate (PAM). (A) PAM exposure significantly increased $V \delta 2$ cell frequency. V $\delta 2$ cells from suppressed $\mathrm{HIV}^{+}$donors $(n=21)$ significantly expanded in response to PAM. Patients treated in the acute infection $(n=9)$ are represented with green triangles and patients treated in chronic infection $(n=12)$ are represented with purple squares. (B) Decreased $V \delta 2$ cell numbers in donors treated in the chronic phase of the infection. HIV-infected donors treated in the chronic phase of the infection ( $n=12$ ) showed significantly reduced number of V $\delta 2$ cells (FDR $P$ $=0.007$ ) compared with those treated in the acute phase of the infection $(n=9)$. After PAM expansion, V $\delta 2$ cells remained significantly lower in donors treated in chronic infection. Mean \pm SEM. Mann-Whitney $U$ test, FDR $P=0.02$. (C) Comparable expansion capacity between patients who initiated ART in the acute or chronic phase of HIV infection. Fold change of $V \delta 2$ cell expansion with PAM was similar in patients treated in the acute and chronic infection. Mean \pm SEM. Mann-Whitney $U$ test.

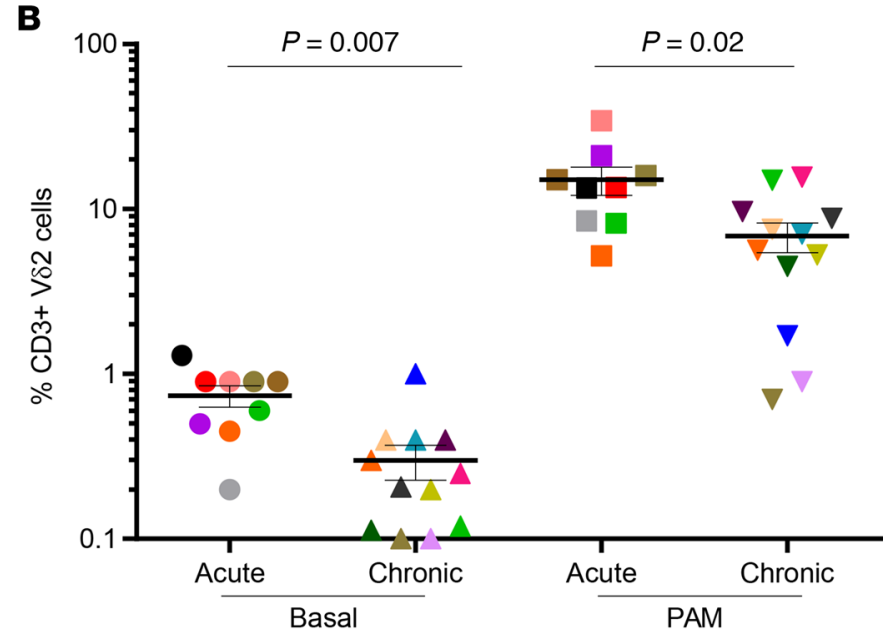

c

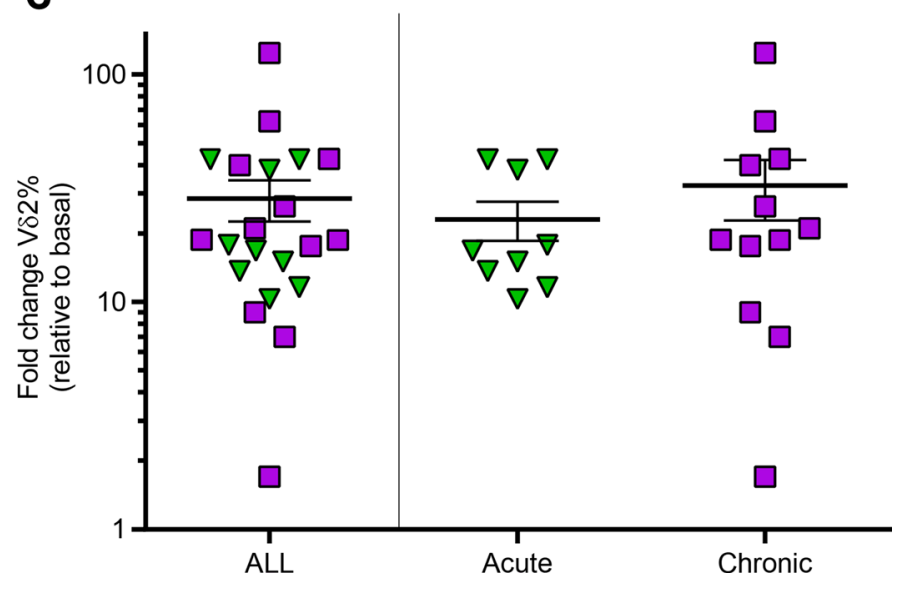

and was variable between subjects (Figure 2A). As we previously reported (24), patients treated in the acute phase of the infection had greater basal V $\delta 2$ cell numbers compared with chronically treated patients $(0.7 \%$ vs. $0.3 \%, P=0.007)$. After expansion, percentages of V $\delta 2$ cells from patients treated in the acute phase of HIV infection were also higher compared with patients treated in the chronic HIV infection (mean 15.0 vs. $6.8, P=0.02$; Figure $2 \mathrm{~B}$ ), but with comparable fold change in expansion ( $P=0.56$, Figure $2 \mathrm{C}$ ). Overall, mean fold change in expansion was 28.4, ranging from 1.7-fold to a more than 124-fold increase. Altogether, our results show that V82 T cells from suppressed HIV-infected donors were successfully expanded in response to ex vivo exposure to PAM and IL-2.

Phenotype of expanded $V \delta 2$ cells. The phenotype of expanded V $\delta 2$ cells after 14 days of exposure to PAM was analyzed in a subgroup of 8 ART-treated, suppressed HIV-infected donors (6 patients treated in chronic infection and 2 patients treated in acute infection) by measuring the expression of markers of memory, cytotoxicity, activation, and immune exhaustion by flow cytometry. V $\delta 2$ memory cell populations were defined as follows: central memory (TCM, $\mathrm{CD}^{2} \mathrm{RA}^{-} \mathrm{CD} 27^{+} \mathrm{CCR} 7^{+}$), transitional memory (TTM, CD45RA $\mathrm{CD} 27^{+} \mathrm{CCR} 7^{-}$), and effector memory (TEM, CD45RA-CD27CCR7-). The majority of expanded V $\delta 2$ cells expressed a TTM phenotype $(65 \%)$, followed by TCM $(23 \%)$ and finally by TEM $(8 \%)$ (Figure 3A). Cytotoxic markers including CD8, CD56, and CD16 were expressed by $37 \%, 32 \%$, and $45 \%$ of expanded V 22 cells, respectively (Figure 3B). Altogether, $50 \%$ of the expanded V $\delta 2$ cells displayed a cytotoxic phenotype $\left(\mathrm{V} \delta 2^{+} \mathrm{CD} 56^{+}\right)$and around $30 \%$ expressed CD16 (suggesting participation in antibody-dependent cell-mediated cytotoxicity). Activation markers CD69, CD25, and HLA-DR were expressed in a mean of $37.2 \%, 14.8 \%$, and $16.6 \%$ V82 cells, respectively. The expression of the exhaustion marker PD-1 was observed in a mean of $19.8 \%$ and CTLA- 4 was observed in $4.3 \%$ of the expanded V 82 cells (Figure 3C).

PAM-expanded VS2 cells inhibit active HIV replication. We previously reported that $\gamma \delta \mathrm{T}$ cells from uninfected donors are potent inhibitors of viral replication in vitro (24). To confirm and extend these results, we used an autologous cellular system to measure the capacity of ex vivo-isolated V $22 \mathrm{~T}$ cells from ART-suppressed HIVinfected donors to inhibit viral replication in $\mathrm{CD}^{+} \mathrm{T}$ cells infected with the JR-CSF strain in vitro. A mean reduction of $85 \%$ in HIV p24 production was detected at a 1:1 effector/target cell ratio, $64 \%$ at 1:10, and $54 \%$ at 1:100 (Figure 4A). HIV p24 production was significantly reduced at 1:1 and 1:10 effector/target cell ratios. 

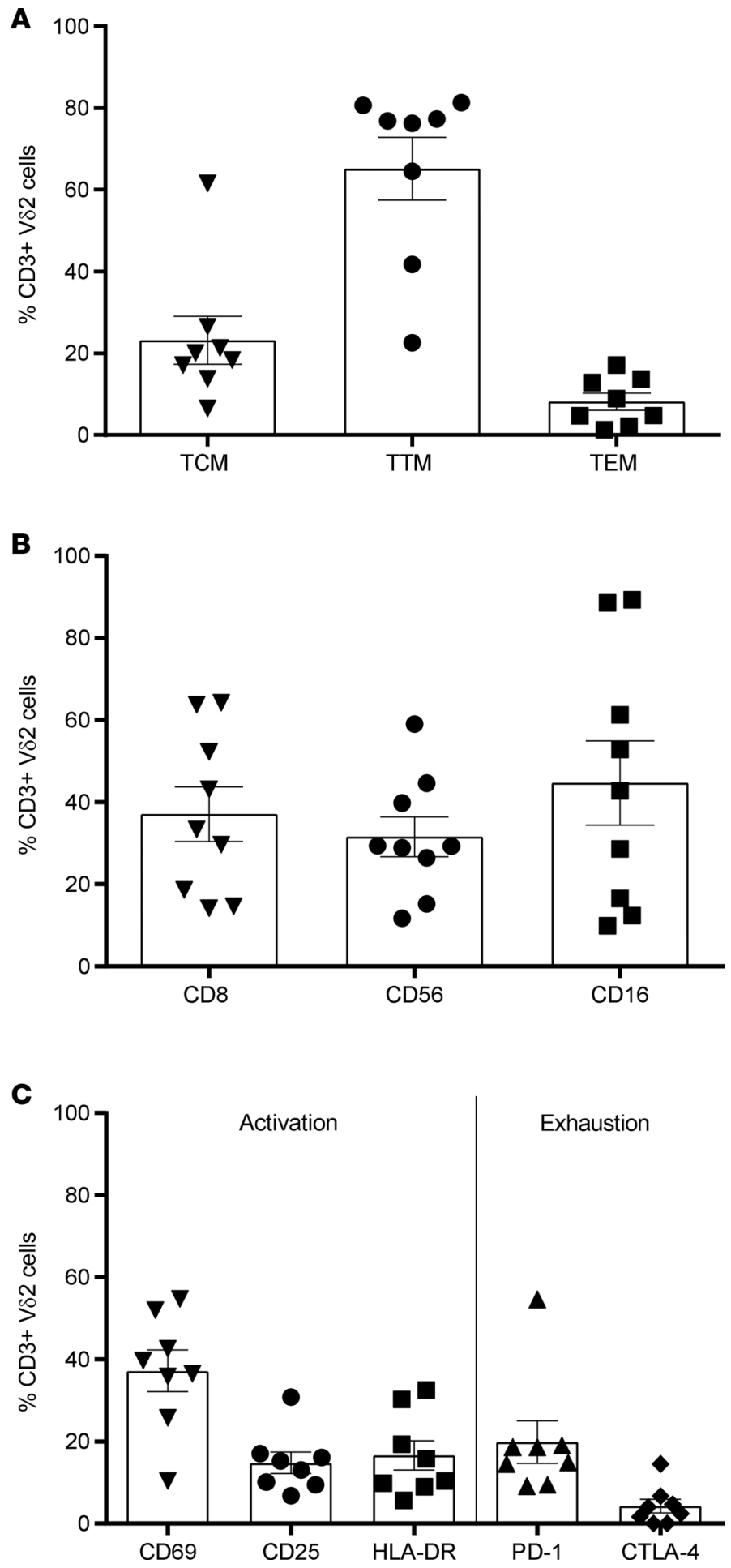

Figure 3. Phenotype of pamidronate-expanded $\mathbf{V} \delta 2$ cells in ARTsuppressed HIV-infected donors. Phenotype of $V \delta 2$ cells was analyzed by flow cytometry in 8 HIV-infected individuals after expansion. Mean \pm SEM is represented. (A) Memory populations defined as central memory (TCM: CD45-CD27 ${ }^{+}$CCR7 ${ }^{+}$), transitional memory (TTM: CD45-CD27+CCR7-), or effector memory (TEM: CD45-CD27-CCR7-). (B) Expression of cytotoxic markers CD8, CD56, and CD16. (C) Expression of activation markers CD69, CD25, and HLA-DR, and exhaustion markers PD-1 and CTLA-4.

Next, we tested the capacity of PAM-expanded V 22 T cells to inhibit HIV replication, using the same functional assays of viral inhibition. Our results show that PAM-expanded V $\delta 2$ cells retain their capacity to significantly inhibit viral replication (Figure 4B and Supplemental Figure 2A). Expanded V $\delta 2$ cells at the $1: 1$ ratio showed a mean inhibition of viral replication of $71 \%$ and $57 \%$ at the $1: 10$ ratio, and $18 \%$ at $1: 100$ To avoid interindividual differences in the comparison of basal and expanded V $\delta 2$ cells, we performed a side-by-side analysis using the same donors. Basal mean viral inhibition mediated by ex vivo V $\delta 2 \mathrm{~T}$ cells was comparable to that measured after expansion with PAM (Figure 4C). We conclude that exposure of V82 T cells to PAM and IL-2 for 14 days does not impair the antiviral function of these cells. Finally, in 4 patients, we compared the inhibition capacity of $V \delta 2$ cells with that of $\mathrm{CD} 8^{+}$ $\mathrm{T}$ cells, showing comparable inhibition capacity between both cell types (Supplemental Figure 2B).

$V \delta 2 \mathrm{~T}$ cells degranulate in the presence of HIV-infected $C D 4^{+}$cells. The ability of V $\delta 2$ cells to target HIV-infected $C D 4^{+} \mathrm{T}$ cells was measured by analyzing CD107a expression. Total CD4 ${ }^{+} \mathrm{T}$ cells from 9 ART-suppressed HIV-infected individuals were isolated and superinfected with the HIV strain JR-CSF to act as targets in cocultures with autologous expanded V $\delta 2$ cells. In preliminary experiments, isolated $\mathrm{CD}^{+}$cells were activated with phytohaemagglutinin (PHA) prior to superinfection with JR-CSF. However, we observed that cytotoxic V 82 cells were activated when $\mathrm{CD} 4^{+}$cells were exposed to PHA, regardless of HIV superinfection (Supplemental Figure 3A). Therefore, we used an alternative protocol to infect isolated $\mathrm{CD} 4^{+}$ cells using polybrene rather than PHA. We detected a significant increase of CD107a expression in expanded V82 cells after coculture with JR-CSF-infected $\mathrm{CD}^{+}{ }^{+}$cells, but not when cultured with $\mathrm{CD}^{+}$cells without superinfection or alone (mean $=13.2 \%, 8.5 \%$, and $8.3 \%$, respectively, $P=0.006$; Figure $5, \mathrm{~A}$ and $\mathrm{B}$ ). Expression of CD107a was also statistically higher in the HIV-CD4+ group activated with PHA (mean $=18.7 \%, P=0.02$ ), but compared with polybrene-infected targets there were not significant differences $(P=$ 0.08). Finally, we determined if the state of HIV infection (acute or chronic) at the time of ART initiation had an impact on $\gamma \delta \mathrm{T}$ cell

effector function. CD107a expression was comparable in cells from acute and chronic treated donors, suggesting that the cytotoxic function of V82 cells may be recovered after ART initiation (Figure 5C). In summary, we demonstrate that expanded V $\delta 2$ cells specifically degranulate in the presence of HIV-infected CD4 ${ }^{+}$cells.

$\gamma \delta \mathrm{T}$ cells can efficiently clear latently HIV-infected cells upon latency disruption. We measured the ability of $\gamma \delta \mathrm{T}$ cells to reduce the recovery of replication-competent HIV after reactivation of resting $\mathrm{CD} 4^{+}\left(\mathrm{r}-\mathrm{CD}^{+}\right)$ $\mathrm{T}$ cells with vorinostat (VOR). We made a modification to the previously reported latency clearance assay protocol used for $\mathrm{CD}^{+} \mathrm{T}$ cells (8), which is itself a modification of viral outgrowth assays. This assay provides evidence of the capacity of effector cells to deplete $\mathrm{r}-\mathrm{CD} 4^{+}$cells producing replication-competent 
A

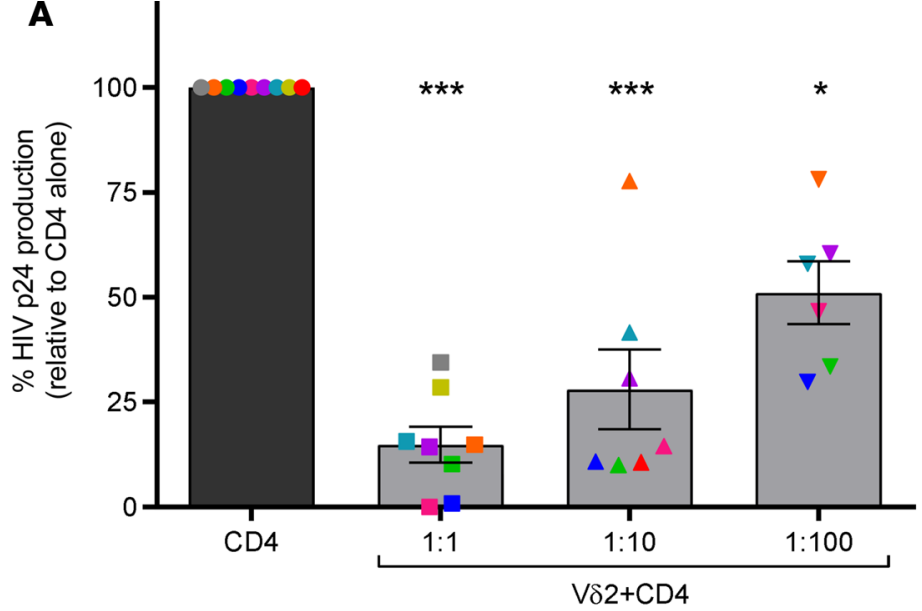

B
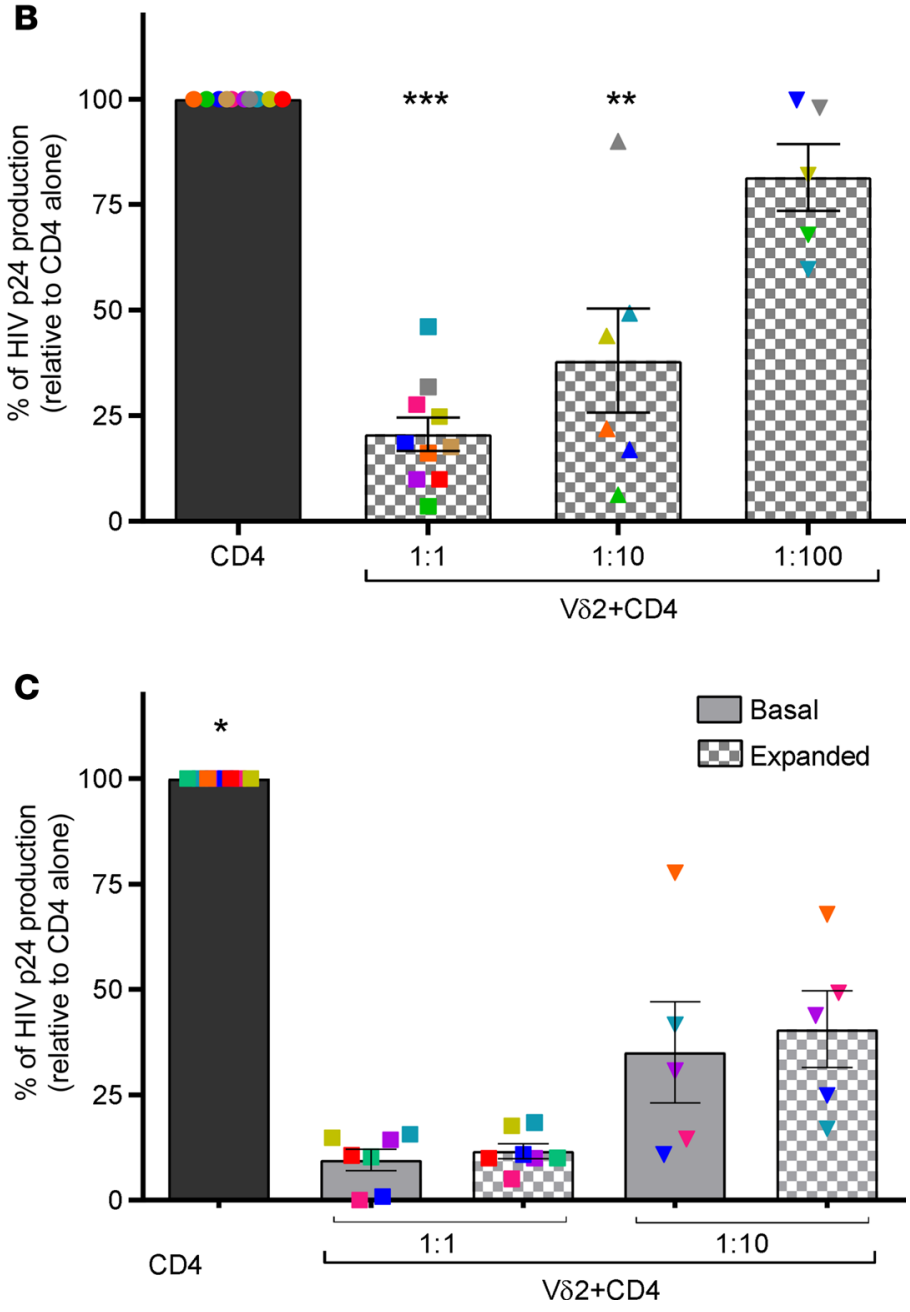

Figure 4. V $\boldsymbol{\delta} \mathbf{2} \mathrm{T}$ cells inhibit active HIV replication. (A) Ex vivo-isolated V $\delta 2 \mathrm{~T}$ cells reduce HIV p24 production. HIV p24 production from autologous superinfected $\mathrm{CD} 4^{+} \mathrm{T}$ cells was significantly reduced in the presence of $\mathrm{V} \delta 2$ ( $n=8$ for the 1:1 effector/target ratio). Bars represent average viral production normalized to the condition where only superinfected CD4+ ${ }^{+}$cells were cultured. Ratios expressed as effector/target cells. (B) Pamidronate-expanded (PAM-expanded) V $\delta 2 \mathrm{~T}$ cells retain their capacity to inhibit viral replication. After 14 days of exposure to PAM, $\gamma \delta$ T cells were cocultured with autologous superinfected $C D 4^{+}$ cells. V $\delta 2$ T cells ( $n=10$ for the 1:1 effector/target ratio) significantly reduced HIV p24 production. (C) Comparable inhibition capacity between basal and PAM-expanded V2 T cells. Data from basal HIV inhibition assays were compared to their respective inhibition capacity after PAM exposure. Inhibition capacity was similar in V $\delta 2$ cells $(n=7$ for the 1:1 effector/target ratio). Mean \pm SEM is represented. MannWhitney $U$ test. ${ }^{*} P<0.05,{ }^{*} P<0.005,{ }^{* *} P<0.0005$.

HIV following latency reversal. Our modification of the assay, which consists of depleting $\gamma \delta \mathrm{T}$ cells after 24 hours of coculture, is critical to allow evaluation of the specific clearance by $\gamma \delta \mathrm{T}$ cells. Adding this modification, we avoid measurement of nonspecific effects on the allogeneic uninfected cells added later for viral outgrowth. Briefly, $\mathrm{r}-\mathrm{CD} 4^{+}$cells from 8 ART-suppressed donors ( 6 treated in chronic infection and 2 treated in acute infection) were isolated, exposed to VOR, and cocultured with or without autologous isolated expanded V $82 \mathrm{~T}$ cells. After 24 hours of culture, $\gamma \delta$ T cells were depleted from the cultures, plated in replicate, and uninfected allogeneic $\mathrm{CD} 4^{+}$cells were added to amplify replication-competent HIV. After 15 out of 19 days of culture, viral outgrowth from $\mathrm{r}-\mathrm{CD} 4^{+}$cells cultured alone was detected in 6 out of 8 HIV-infected donors, as measured by the number of HIV p24-positive wells. Interestingly, when $\gamma \delta$ T cells were present in the coculture system, no virus was recovered in 4 donors, and viral recovery was reduced from 5 to 2 , and 4 to 3 culture wells in the other 2 participants (Figure 6). These results demonstrate that expanded $\mathrm{V} \delta 2 \mathrm{~T}$ cells can clear latently infected cells at the time of latency disruption by VOR.

\section{Discussion}

In this study, we assessed the prevalence, phenotype, function, and ex vivo expansion capability of $\gamma \delta \mathrm{T}$ cells from ARTsuppressed HIV-infected individuals. We demonstrate the cytotoxic capabilities of these cells, as measured by their ability to kill autologous HIV-infected $\mathrm{CD} 4^{+} \mathrm{T}$ cells. In addition, V $\delta 2 \mathrm{~T}$ cells were able to expand up to 120 -fold in response to PAM/IL-2 ex vivo and reduce viral replication up to $80 \%$ in autologous coculture systems. Overall, our results support the important finding that $\gamma \delta \mathrm{T}$ cells possess antiviral capabilities that are maintained in virologically suppressed individuals.

Further, such antiviral $\gamma \delta \mathrm{T}$ cells can be expanded ex vivo to target latently infected cells induced to express HIV. The present work constitutes the first proof-of-concept to our knowledge showing that in the context of durable suppression of HIV infection, $\gamma \delta \mathrm{T}$ cells are capable of eliminating HIV-infected targets, suggesting that $\gamma \delta \mathrm{T}$ cells should be explored as a novel effector population to clear HIV infection from latent and active reservoirs.

As previously reported for the N-BP Zol (21), we show that PAM induces expansion of V $\delta 2$ cells in HIV-infected individuals. Although V2 cell numbers were higher in patients treated in the acute phase 
A

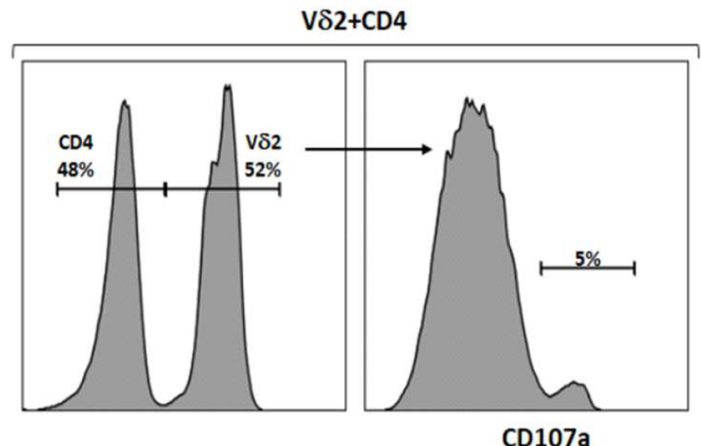

VS2+JR-CSF-CD4

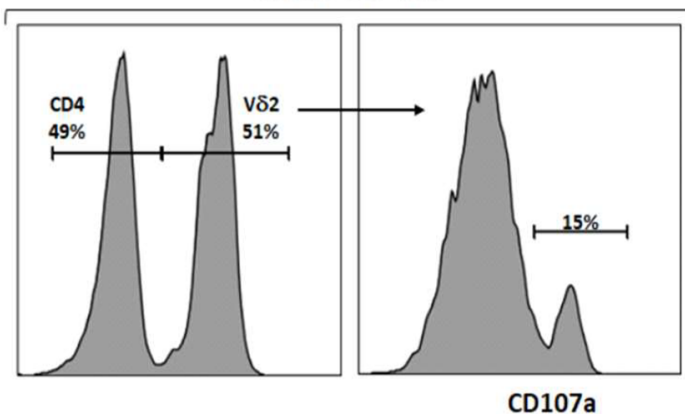

B

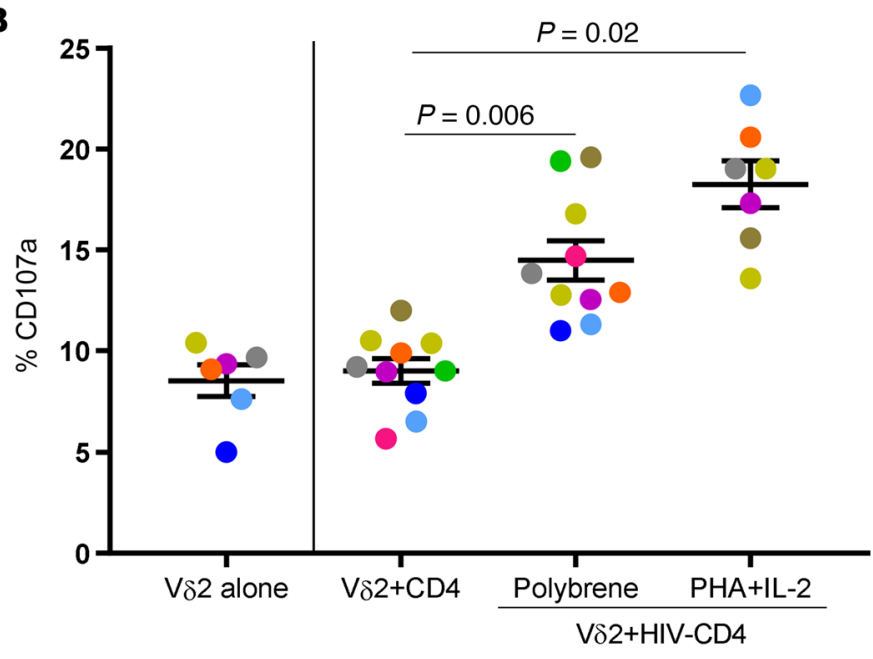

C

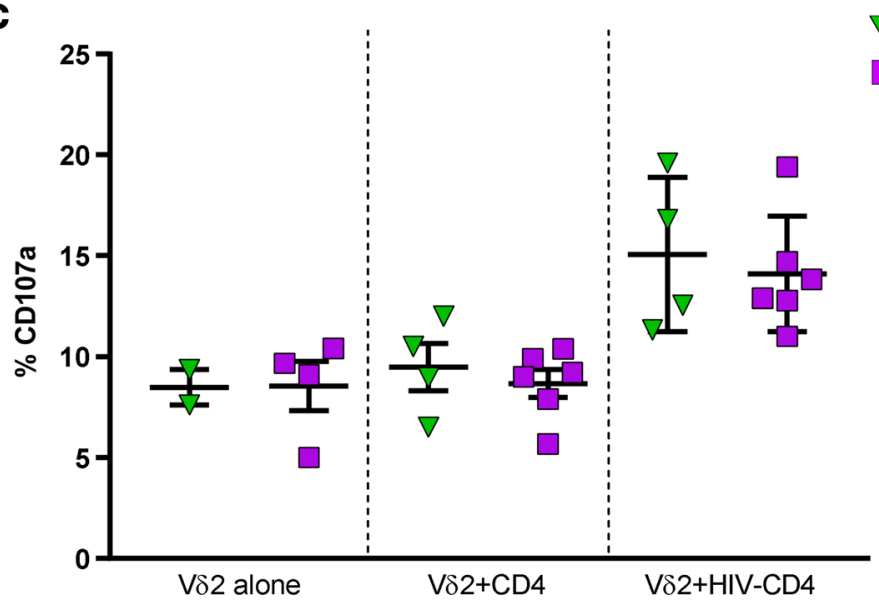

Figure 5. V $\mathbf{2} 2$ cells degranulate in the presence of autologous HIV-infected CD4 ${ }^{+} \mathbf{T}$ cells. (A) Flow cytometry plots showing an example of CD107a detection in cocultures of expanded V $\delta 2$ cells with autologous CD4+ cells (left) and with autologous JR-CSF-superinfected CD4+ cells (right). (B) Greater CD107a production in the presence of HIV-infected cells. CD107a production was statistically higher when V $\delta 2$ cells were cocultured with HIV-superinfected CD4 $4^{+}$cells compared with cocultures of autologous isolated CD4 ${ }^{+}$cells (FDR $\left.P=0.006\right)$. CD107a production was the highest when V $\delta 2$ cells were cocultured with PHA-activated, HIV-superinfected CD4+ cells (FDR $P=0.02$ ) but without statistical differences compared with cells infected using polybrene. Mean \pm SEM is represented. $P=0.08$, Wilcoxon's matched-pairs signed-rank test. (C) Comparable degranulation capacity of $V \delta 2$ cells between donors treated in acute and chronic HIV infection. CD107a production was not statistically different between acute and chronic patients. Both groups of patients showed statistically higher CD107a expression in cocultures of V $\delta 2$ cells and superinfected CD4 ${ }^{+}$target cells than in cultures of $\mathrm{V} \delta 2$ cells cocultured with ex vivo-isolated CD4+ cells. Effector/target ratio (1:1). Mann-Whitney $U$ test. 


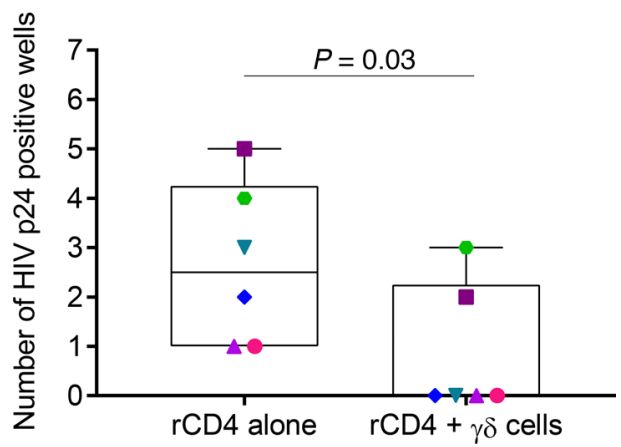

Figure 6. $\boldsymbol{\gamma} \boldsymbol{\delta} \mathrm{T}$ cells clear latently infected cells after latency reversal with vorinostat (VOR). Isolated resting $\mathrm{CD4}^{+}\left(\mathrm{r}-\mathrm{CD} 4^{+}\right)$cells from ART-suppressed HIV-infected donors were reactivated with $0.5 \mu \mathrm{M}$ VOR. After washing, $r-C D 4^{+}$cells were cultured alone or with $\gamma \delta \mathrm{T}$ cells, which were removed from the culture after 24 hours. The same number of replicate cultures of $1 \times$ $10^{6}$ from each condition were then cultured in parallel for 19 days. VOR efficiently reactivated latent HIV in 6 (represented in the graph) of the 8 patients analyzed in the condition where $\mathrm{r}-\mathrm{CD} 4^{+}$cells were cultured alone. Frequency of HIV recovery (number of positive wells for HIV p24 measured by ELISA) decreased significantly $(P=0.03$, Wilcoxon's signed-rank test) in cultures of $\mathrm{r}-\mathrm{CD} 4^{+}$cells in the presence of $\gamma \delta$ T cells, demonstrating that $\gamma \delta$ T cells can recognize and clear latently HIV-infected cells upon latency reversal.

of the infection compared with chronic infection, expansion capacity was comparable between both groups. Unfortunately, due to the difficulty in recruiting women (31), our study only included men. However, we acknowledge that gender differences may exist, given controversial findings in previous work according to $\gamma \delta \mathrm{T}$ cell frequencies (32-34), that may potentially translate into functional differences. Current work is focused towards this end. PAM-expanded V82 cells were mostly in a transitional memory state, characterized by elevated IFN- $\gamma$ production $(35,36)$. This phenotype has also been reported both after in vitro exposure to different N-BPs (37) and in vivo after Zol treatment in cancer patients (38). In addition, cytotoxic phenotype in $\gamma \delta \mathrm{T}$ cells has been associated with the expression of CD16, CD56, and CD8 (34). Frequencies of expression of cytotoxic markers in expanded V82 cells in our study are in accordance with those reported in cancer and HIV settings $(25,39,40)$. Finally, although PD-1 and CTLA-4 are well-known markers of immune-exhausted CD8 ${ }^{+} \mathrm{T}$ cells (41), the significance of their expression on expanded V $\delta 2$ cells remains to be elucidated (41).

PAM-expanded V $\delta 2$ cells showed increased CD107a production after coculture with autologous HIVsuperinfected $\mathrm{CD}^{+}$cell targets compared with cocultures of autologous $\mathrm{CD} 4^{+}$cells without superinfection, demonstrating specific degranulation triggered by HIV infection. CD107a production, although statistically significant, was not very high, suggesting the involvement of other pathways in HIV recognition (42). In addition, as only a fraction of the $\mathrm{CD} 4^{+}$cells used as targets may be infected, CD107a production may be potentially diminished compared with an assay where $100 \%$ of targets were activating $\gamma \delta \mathrm{T}$ cells. Antigen recognition by the $\gamma$ TCR is generally not restricted to major MHC molecules $(14,15)$, although $\gamma \delta \mathrm{T}$ cell recognition of peptides loaded on MHC molecules has been reported (43). Interestingly, our MHC blocking experiments showed a moderate decrease in CD107a expression in V82 cells that needs to be further investigated (Supplemental Figure 3). Our study also highlights the importance of carefully controlling for external factors in culture systems that might provide confounding results - such as the use of PHA to activate prior to infection, as cytotoxic V $\delta 2$ cells were activated by cells treated with PHA independently of HIV infection. Even when isolated $\mathrm{CD} 4^{+}$cells were allowed to rest for 6 to 7 days after PHA activation, V82 cells showed increased CD107a production, suggesting that PHA binding to glycosylated surface proteins produces longterm, and perhaps irreversible, changes at the surface of cells that are recognized by $\gamma \delta \mathrm{T}$ cells.

To further analyze the role of $\gamma \delta \mathrm{T}$ cells in a context more relevant to HIV eradication in vivo, we performed a modification of the previously reported latency clearance assay $(8,44)$. In this assay, the capacity of effector cells to recognize latent HIV reactivated by LRAs was evaluated. In our modified assay, we depleted $\gamma \delta \mathrm{T}$ cells from the coculture before the addition of uninfected target cells to ensure specific clearance of $\mathrm{r}-\mathrm{CD} 4^{+}$infected cells. Our results demonstrate that $\gamma \delta \mathrm{T}$ cells reduce viral recovery following latency reversal of $\mathrm{r}-\mathrm{CD} 4^{+}$cells with VOR. Immunotherapy with ex vivo-expanded $\gamma \delta$ cells has been used in oncology with little toxicity and overall good tolerability (27). Of great significance for future strategies aimed to cure HIV, the use of haploidentical expanded V $\delta 2$ cells from relatives in adoptive transfer strategies has been successful $(45,46)$. This type of intervention may be valuable for future use in HIV eradication strategies, as $\gamma \delta \mathrm{T}$ cells can harbor replication-competent HIV (24). In this regard, supernatants from expansions showed a consistent negative HIV p24 detection. However, even if cells are expanded in the presence of antiretrovirals avoiding new rounds of infection, we still do not completely understand the overall importance of defective viruses that may be accumulating, and their modulating effector responses (47).

In summary, $\gamma \delta \mathrm{T}$ cells from HIV-infected individuals retain their functionality after expansion and constitute an attractive immunotherapeutic alternative or complementary tool to current approaches aimed 
to cure HIV. Our work has opened potentially novel and intriguing questions regarding the basic biology, function, and specificities of $\gamma \delta \mathrm{T}$ cells. Here, we show proof-of-concept of the potential clinical use of $\gamma \delta \mathrm{T}$ cells in cellular therapy strategies for HIV cure.

\section{Methods}

Study participants. All HIV-infected donors included in this study were on ART and virologically suppressed $(<50$ copies $/ \mathrm{ml})$ for at least 1 year prior to study. Characteristics and inclusion criteria of these donors have been previously described $(24,48)$. HIV-infected donors treated in the acute phase of HIV infection started therapy within 45 days of the estimated date of infection. Buffy coats from uninfected donor volunteers were obtained from the New York Blood Center.

Isolation of cell populations. PBMCs from HIV-infected individuals were isolated from leukapheresis products, and cells from uninfected individuals were isolated from buffy coats, all by Ficoll-gradient centrifugation. V 82 cells and CD8 ${ }^{+} \mathrm{T}$ cells were isolated by fluorescence-activated cell sorting (FACS) using a FACSAria II (BD). PBMCs were stained with monoclonal antibodies against CD3 (clone SK7), V82 (clone B6), CD4 (clone SK3), and CD8 (clone SK1) (all from Biolegend). V2 T cells were defined as $\mathrm{CD} 3^{+} \mathrm{V} \delta 2^{+}$ and $\mathrm{CD}^{+} \mathrm{T}$ cells were defined by $\mathrm{CD}^{+} \mathrm{V} \delta 1^{-} \mathrm{V} \delta 2^{-} \mathrm{CD} 4^{-} \mathrm{CD} 8^{+} . \mathrm{CD}^{+} \mathrm{T}$ cells were isolated from the same donor using a commercially available enrichment kit that contains antibodies against CD8, CD14, CD16, CD19, CD20, CD36, CD56, CD66b, CD123, TCR- $\gamma / \delta$, and glycophorin A (StemCell Technologies). $\mathrm{r}-\mathrm{CD} 4^{+}$cells were isolated using a custom StemCell Technologies cocktail that contained antibodies against the following proteins: CD8, CD14, CD16, CD19, CD20, CD36, CD56, CD123, $\gamma \delta T C R$, glycophorin A, CD66b, CD25, HLADR, and CD69. Purity of the FACS-isolated populations and resting CD4 $4^{+}$cells was greater than $99 \%$, and magnetically isolated CD4 ${ }^{+} \mathrm{T}$ cells showed a purity of greater than $96 \%$.

Expansion of V 22 cells. To compare V 22 cell response to pyrophosphates and N-BP from HIV-infected and uninfected donors, $1 \times 10^{6} \mathrm{PBMCs}$ were incubated in the presence of $100 \mathrm{nM} \mathrm{HMBPP}$ (provided by H. Jomaa, Justus-Liebig University, Giessen, Germany) and $100 \mathrm{U} / \mathrm{ml} \mathrm{IL-2,} \mathrm{or} 25$ g/ml PAM and $100 \mathrm{U} / \mathrm{ml} \mathrm{IL}-2$, or $100 \mathrm{U} / \mathrm{ml}$ IL-2 alone. After 6 days, cells were stained with monoclonal antibodies against CD3 and V2 to analyze $\gamma \delta \mathrm{T}$ cell frequency by flow cytometry. Briefly, cells were harvested, washed, resuspended in staining buffer, incubated on ice in the dark for 20 minutes and finally washed and resuspended in $2 \%$ paraformaldehyde solution. Acquisition and analysis was performed on the Attune NxT flow cytometer (Applied Biosystems).

Fifty to 70 million PBMCs from HIV-infected individuals were incubated in complete IMDM containing antiretrovirals (10 mM raltegravir and either $10 \mathrm{mM}$ abacavir or $1 \mu \mathrm{M}$ efavirenz, depending on the patient's regimen), $25 \mu \mathrm{g} / \mathrm{ml}$ pamidronate (PAM, Sigma-Aldrich), and $200 \mathrm{U} / \mathrm{ml}$ IL-2 during 14 days. Every 3-4 days, medium containing $100 \mathrm{U} / \mathrm{ml} \mathrm{IL}-2$ was refreshed. HIV p24 quantification (ABL, Inc.) at days 7 and 14 was consistently under the limit of detection of the assay.

Phenotypic analysis of expanded V 2 cells. At day 14 of expansion, an aliquot of PBMCs was harvested, washed, and resuspended in staining buffer to analyze the expression of different markers within expanded V82 cells by flow cytometry. (a) Memory: CD45RA (clone HI100), CD27 (clone MT271), and CCR7 (clone G043H7); (b) cytotoxic: CD8 (clone SK1), CD56 (clone 5.1H11), and CD16 (clone 3G8); (c) activation: CD69 (clone FN50), CD25 (clone BC96), and HLA-DR (clone L243); and exhaustion: PD-1 (clone EH12.2H7) and CTLA-4 (clone BNI3). Cells were stained for 20 minutes on ice in the dark, washed, fixed in a $2 \%$ paraformaldehyde solution, acquired on a FACSAria II, and analyzed using FlowJo v.10.1 (Tree Star).

HIV infection of isolated $C D 4^{+} \mathrm{T}$ cells. Isolated $\mathrm{CD}^{+} \mathrm{T}$ cells from HIV-infected donors were superinfected with the viral strain JR-CSF using 2 different approaches. (a) CD4 ${ }^{+}$cells were activated with 4 $\mu \mathrm{g} / \mathrm{ml} \mathrm{PHA}$ and $100 \mathrm{U} / \mathrm{ml} \mathrm{IL}-2$ for 24 hours, washed twice, and superinfected by spinoculation at 2,500 rpm for 2 hours. Cells were then extensively washed to remove free virions and then used for further experiments. (b) Isolated CD4 ${ }^{+}$cells were spinoculated at 2,500 rpm for 4 hours in the presence of $8 \mu \mathrm{g} /$ $\mathrm{ml}$ polybrene. Cells were resuspended in complete media containing $20 \mathrm{U} / \mathrm{ml} \mathrm{IL}-2$ and without washing the virus were further cultured for 7 days. Then, cells were extensively washed, resuspended in suitable media and experiments were performed. As a control, isolated $\mathrm{CD}^{+}{ }^{+}$cells were mock infected following the same 2 approaches. Level of infection of isolated $\mathrm{CD} 4^{+}$cells was routinely tested, quantifying HIV p24 production in culture supernatants by ELISA. Results showed a consistent efficacy of infection with a mean of $264.1 \mathrm{ng} / \mathrm{ml}$ for cells activated with PHA and $128.9 \mathrm{ng} / \mathrm{ml}$ for cells infected with polybrene. HIV p24 production was below the limit of detection of the assay for non-superinfected $\mathrm{CD}^{+} \mathrm{T}$ cells. 
Viral inhibition assays. Viral inhibition assays using V82 cells from HIV-infected individuals as effectors were performed as previously described for uninfected individuals (24), with some modifications. Fifty thousand infected $\mathrm{CD}^{+} \mathrm{T}$ cells were cocultured in triplicate at different effector/target ratios of 1:1, 1:10, and 1:100, unless otherwise noted. Infected $\mathrm{CD}^{+}$cells alone were also cultured in triplicate as control for HIV production. In some experiments, ex vivo-isolated CD8 ${ }^{+} \mathrm{T}$ cells were used as effector cells. Supernatants were harvested at day 7 and stored at $-20^{\circ} \mathrm{C}$ until HIVp24 ELISA quantification (ABL, Inc.) was performed. Results are expressed as percentage of viral inhibition normalized to HIV p24 production when target CD4 ${ }^{+} \mathrm{T}$ cells were cultured alone.

Degranulation assays. CD107a was used as a functional marker of cytotoxicity. FACS-isolated, expanded V82 T cells were cocultured with JR-CSF-infected autologous CD4 ${ }^{+}$cells as targets. CD4 ${ }^{+} \mathrm{T}$ cells were infected following the 2 different approaches described above. At least 100,000 effector cells were cocultured at a 1:1 ratio with $\mathrm{CD}^{+}$target cells in 96-well plates for 5 hours in the presence of GolgiStop (BD) and an anti-CD107a monoclonal antibody (clone H4A3, BD). In some experiments, MHC expression was blocked by incubating the cells with a pan-HLA monoclonal antibody (clone W6/32, Biolegend). Cells were then harvested, washed with staining buffer, stained with V82-FITC (Biolegend) for 20 minutes on ice in the dark, washed twice, resuspended in staining buffer, and analyzed in the Attune Acoustic Focusing Cytometer (Applied Biosystems).

Assays to analyze clearance of latent HIV after reactivation. These experiments are based on the latency clearance assay reported for $\mathrm{CD}^{+} \mathrm{T}$ cells (8) and more recently for NK cells (44). Briefly, $\mathrm{r}-\mathrm{CD}^{+}{ }^{+}$cells from HIV-infected donors were isolated as described above, and exposed to $0.5 \mu \mathrm{M}$ VOR for 18 hours. After washing, $\mathrm{r}-\mathrm{CD} 4{ }^{+}$cells were split into 2 conditions, one cultured alone and the other cocultured with isolated expanded $\gamma \delta \mathrm{T}$ cells at a 2:10 ratio $\left(\gamma \delta\right.$ cell/r-CD4 ${ }^{+}$cell). After 24 hours of culture, $\gamma \delta$ cells were removed using a depletion magnetic bead-based kit according to the manufacturer's instructions (Miltenyi Biotec). The same treatment was applied to both conditions in parallel. This $\gamma \delta \mathrm{T}$ cell depletion after the initial 24 hours of culture constitutes our modification of the original latency clearance assay reported for $\mathrm{CD}^{+} \mathrm{T}$ cells, providing definitive proof that the effect observed at the end of the culture is due to clearance of the reactivated $\mathrm{r}-\mathrm{CD} 4^{+}$cells by $\delta$ T cells. After depletion, $\mathrm{r}-\mathrm{CD} 4^{+}$cells were plated at 1 million cells/well. The same number of wells (from 7 to 23 replicates) were assayed for the condition of r-CD4 ${ }^{+}$cells alone and $\mathrm{r}-\mathrm{CD} 4^{+}$cells cocultured with $\gamma \delta$ T cells. As target cells to amplify viral signal, we added isolated, PHAactivated total $\mathrm{CD}^{+}$cells from uninfected donors at days 3 and 8 of culture. Supernatants were harvested at day 15 and 19 by HIV p24 ELISA. Depletion of $\gamma \delta$ T cells after coculture was analyzed by flow cytometry, showing greater than $99.9 \%$ efficacy. Results are expressed as number of positive wells for HIV p24, comparing cultures of $\mathrm{r}-\mathrm{CD} 4^{+}$cells with and without $\gamma \delta$ T cells.

Study approval. All patients provided written informed consent prior to inclusion in the study, and studies were approved by the University of North Carolina (UNC) Institutional Review Board.

Statistics. Nonparametric tests were used. Comparisons between different groups were performed using the Mann-Whitney $U$ test and repeated measures within same groups were analyzed using Wilcoxon's matched-pairs signed-rank test. Where indicated, multiple comparisons were accounted for using FDRadjusted $P$ values (49). Statistical significance was considered for $P<0.05$.

\section{Author contributions}

DMM and NSS conceptualized the study, provided resources, and acquired funding. CG and NSS developed the methodology. CG, MLC, CPW, and NSS performed the investigation. CG, DMM, and NSS wrote the draft of the manuscript. MH and NSS performed statistical analyses. NSS supervised the study.

\section{Acknowledgments}

We thank Jennifer Kirchherr, Erin Stuelke, Katherine Sholtis, and Brigitte Allard for technical support. We thank Cynthia Gay, Joann Kuruc, and Caroline Baker for donor recruitment and clinical management. The authors thank Nancy Fisher and Evan Trudeau from the UNC Flow Cytometry Core. We would like to thank Nancie Archin and David Favre for critical reading the manuscript. Finally, we are grateful for the contributions of the $\mathrm{HIV}^{+}$participants. This work was supported by NIH R01 AI12509-01A1 to N. Soriano-Sarabia. Research reported in this publication was supported, in part, by CARE, a Martin Delaney Collaboratory, the National Institute of Allergy and Infectious Diseases (NIAID), National Institute of Neurological Disorders and Stroke (NINDS), National Institute on Drug Abuse (NIDA), and the National Institute of Mental Health (NIMH) of the NIH, grant number 1UM1AI126619-01. The content is solely 
the responsibility of the authors and does not necessarily represent the official views of the NIH. The UNC Flow Cytometry Core Facility is supported in part by P30 CA016086 Cancer Center Core Support Grant to the UNC Lineberger Comprehensive Cancer Center, which is supported in part by the North Carolina Biotech Center Institutional Support Grant 2012-IDG-1006. This research was partially supported by the Center for AIDS Research (CFAR) P30 AI50410.

Address correspondence to: Natalia Soriano-Sarabia, 2013 Genetic Medicine Building, 120 Mason Farm Road, CB 7042, University of North Carolina at Chapel Hill, Chapel Hill, North Carolina 27599-7042, USA. Phone: 919.966.8415; Email: natalia_soriano@med.unc.edu.

1. Archin NM, Sung JM, Garrido C, Soriano-Sarabia N, Margolis DM. Eradicating HIV-1 infection: seeking to clear a persistent pathogen. Nat Rev Microbiol. 2014;12(11):750-764.

2. Chun TW, et al. Quantification of latent tissue reservoirs and total body viral load in HIV-1 infection. Nature. 1997;387(6629):183-188.

3. Wong JK, et al. Recovery of replication-competent HIV despite prolonged suppression of plasma viremia. Science. 1997;278(5341):1291-1295.

4. Finzi D, et al. Latent infection of $\mathrm{CD}^{+} \mathrm{T}$ cells provides a mechanism for lifelong persistence of HIV-1, even in patients on effective combination therapy. Nat Med. 1999;5(5):512-517.

5. Siliciano JD, Siliciano RF. Recent developments in the effort to cure HIV infection: going beyond $\mathrm{N}=1$. J Clin Invest. 2016;126(2):409-414.

6. Margolis DM, Garcia JV, Hazuda DJ, Haynes BF. Latency reversal and viral clearance to cure HIV-1. Science. 2016;353(6297):aaf6517.

7. Deng K, et al. Broad CTL response is required to clear latent HIV-1 due to dominance of escape mutations. Nature. 2015;517(7534):381-385.

8. Sung JA, et al. Expanded cytotoxic T-cell lymphocytes target the latent HIV reservoir. J Infect Dis. 2015;212(2):258-263.

9. Day CL, et al. PD-1 expression on HIV-specific T cells is associated with T-cell exhaustion and disease progression. Nature. 2006;443(7109):350-354.

10. Shan L, et al. Stimulation of HIV-1-specific cytolytic T lymphocytes facilitates elimination of latent viral reservoir after virus reactivation. Immunity. 2012;36(3):491-501.

11. Morita CT, Jin C, Sarikonda G, Wang H. Nonpeptide antigens, presentation mechanisms, and immunological memory of human Vgamma2Vdelta2 T cells: discriminating friend from foe through the recognition of prenyl pyrophosphate antigens. Immunol Rev. 2007;215:59-76.

12. Jomaa $\mathrm{H}$, et al. Inhibitors of the nonmevalonate pathway of isoprenoid biosynthesis as antimalarial drugs. Science. 1999;285(5433):1573-1576.

13. Hintz M, et al. Identification of (E)-4-hydroxy-3-methyl-but-2-enyl pyrophosphate as a major activator for human gammadelta T cells in Escherichia coli. FEBS Lett. 2001;509(2):317-322.

14. Tanaka Y, Morita CT, Tanaka Y, Nieves E, Brenner MB, Bloom BR. Natural and synthetic non-peptide antigens recognized by human gamma delta T cells. Nature. 1995;375(6527):155-158

15. Vavassori S, et al. Butyrophilin 3A1 binds phosphorylated antigens and stimulates human $\gamma \delta \mathrm{T}$ cells. Nat Immunol. 2013;14(9):908-916.

16. Wang $\mathrm{H}$, et al. Indirect stimulation of human $\mathrm{V} \gamma 2 \mathrm{~V} \delta 2 \mathrm{~T}$ cells through alterations in isoprenoid metabolism. J Immunol. 2011;187(10):5099-5113.

17. Poccia F, et al. Phosphoantigen-reactive Vgamma9Vdelta2 T lymphocytes suppress in vitro human immunodeficiency virus type 1 replication by cell-released antiviral factors including CC chemokines. J Infect Dis. 1999;180(3):858-861.

18. Lo Presti E, Dieli F, Meraviglia S. Tumor-Infiltrating $\gamma \delta$ T lymphocytes: Pathogenic role, clinical significance, and differential programing in the tumor microenvironment. Front Immunol. 2014;5:607.

19. Silva-Santos B, Serre K, Norell H. $\gamma \delta$ T cells in cancer. Nat Rev Immunol. 2015;15(11):683-691.

20. Wallace M, Bartz SR, Chang WL, Mackenzie DA, Pauza CD, Malkovsky M. Gamma delta T lymphocyte responses to HIV. Clin Exp Immunol. 1996;103(2):177-184.

21. Poonia B, Pauza CD. Gamma delta T cells from $\mathrm{HIV}^{+}$donors can be expanded in vitro by zoledronate/interleukin-2 to become cytotoxic effectors for antibody-dependent cellular cytotoxicity. Cytotherapy. 2012;14(2):173-181.

22. Fausther-Bovendo H, Wauquier N, Cherfils-Vicini J, Cremer I, Debré P, Vieillard V. NKG2C is a major triggering receptor involved in the V[delta]1 T cell-mediated cytotoxicity against HIV-infected CD4 T cells. AIDS. 2008;22(2):217-226.

23. Hudspeth K, et al. Engagement of NKp30 on V $\delta 1 \mathrm{~T}$ cells induces the production of CCL3, CCL4, and CCL5 and suppresses HIV-1 replication. Blood. 2012;119(17):4013-4016.

24. Soriano-Sarabia N, et al. Peripheral V $\gamma 9 \mathrm{~V} \delta 2 \mathrm{~T}$ cells are a novel reservoir of latent HIV infection. PLoS Pathog. 2015;11(10):e1005201.

25. Lafont V, et al. Plasticity of $\gamma \delta$ T cells: Impact on the anti-tumor response. Front Immunol. 2014;5:622.

26. Poccia F, et al. Zoledronic acid and interleukin-2 treatment improves immunocompetence in HIV-infected persons by activating Vgamma9Vdelta2 T cells. AIDS. 2009;23(5):555-565.

27. Kobayashi H, Tanaka Y. $\gamma \delta$ T cell immunotherapy-A review. Pharmaceuticals (Basel). 2015;8(1):40-61.

28. Zou C, Zhao P, Xiao Z, Han X, Fu F, Fu L. $\gamma \delta$ T cells in cancer immunotherapy. Oncotarget. 2017;8(5):8900-8909.

29. Van Acker HH, Anguille S, Willemen Y, Smits EL, Van Tendeloo VF. Bisphosphonates for cancer treatment: Mechanisms of action and lessons from clinical trials. Pharmacol Ther. 2016;158:24-40. 
30. Poccia F, et al. Peripheral V gamma 9/V delta $2 \mathrm{~T}$ cell deletion and anergy to nonpeptidic mycobacterial antigens in asymptomatic HIV-1-infected persons. J Immunol. 1996;157(1):449-461.

31. Loutfy MR, et al. Recruitment of HIV-positive women in research: discussing barriers, facilitators, and research personnel's knowledge. Open AIDS J. 2014;8:58-65.

32. Caccamo N, Dieli F, Wesch D, Jomaa H, Eberl M. Sex-specific phenotypical and functional differences in peripheral human Vgamma9/Vdelta2 T cells. J Leukoc Biol. 2006;79(4):663-666.

33. Cairo C, et al. Impact of age, gender, and race on circulating $\gamma \delta$ T cells. Hum Immunol. 2010;71(10):968-975.

34. Ryan PL, et al. Heterogeneous yet stable V $\delta 2(+)$ T-cell profiles define distinct cytotoxic effector potentials in healthy human individuals. Proc Natl Acad Sci USA. 2016;113(50):14378-14383.

35. Dieli F, et al. Differentiation of effector/memory Vdelta2 T cells and migratory routes in lymph nodes or inflammatory sites J Exp Med. 2003;198(3):391-397.

36. Fritsch RD, Shen X, Sims GP, Hathcock KS, Hodes RJ, Lipsky PE. Stepwise differentiation of CD4 memory T cells defined by expression of CCR7 and CD27. J Immunol. 2005;175(10):6489-6497.

37. Ferlazzo V, et al. In vitro effects of aminobisphosphonates on Vgamma9Vdelta2 $\mathrm{T}$ cell activation and differentiation. Int J Immunopathol Pharmacol. 2006;19(2):309-317.

38. Dieli F, et al. Induction of gammadelta T-lymphocyte effector functions by bisphosphonate zoledronic acid in cancer patients in vivo. Blood. 2003;102(6):2310-2311.

39. Alexander AA, et al. Isopentenyl pyrophosphate-activated $\mathrm{CD} 56^{+}$\{gamma\} \{delta\} T lymphocytes display potent antitumor activity toward human squamous cell carcinoma. Clin Cancer Res. 2008;14(13):4232-4240.

40. Urban EM, Li H, Armstrong C, Focaccetti C, Cairo C, Pauza CD. Control of CD56 expression and tumor cell cytotoxicity in human Vgamma2Vdelta2 T cells. BMC Immunol. 2009;10:50.

41. Teigler JE, et al. Differential inhibitory receptor expression on T cells delineates functional capacities in chronic viral infection J Virol. 2017;91(23):e01263-17.

42. Pauza CD, Poonia B, Li H, Cairo C, Chaudhry S. $\gamma \delta$ T cells in HIV disease: Past, present, and future. Front Immunol. 2014;5:687.

43. Born WK, et al. Peptide antigens for gamma/delta T cells. Cell Mol Life Sci. 2011;68(14):2335-2343.

44. Garrido C, et al. IL-15 stimulated natural killer cells clear HIV-1 infected cells following latency reversal ex vivo [published online ahead of print March 28, 2018]. J Virol. https://www.https://doi.org/10.1128/JVI.00235-18.

45. Wilhelm M, et al. Successful adoptive transfer and in vivo expansion of haploidentical $\gamma \delta \mathrm{T}$ cells. J Transl Med. 2014;12:45.

46. Norell H, Moretta A, Silva-Santos B, Moretta L. At the bench: Preclinical rationale for exploiting NK cells and $\gamma \delta$ T lymphocytes for the treatment of high-risk leukemias. J Leukoc Biol. 2013;94(6):1123-1139.

47. Bruner KM, et al. Defective proviruses rapidly accumulate during acute HIV-1 infection. Nat Med. 2016;22(9):1043-1049.

48. Archin NM, et al. Immediate antiviral therapy appears to restrict resting CD4 ${ }^{+}$cell HIV-1 infection without accelerating the decay of latent infection. Proc Natl Acad Sci USA. 2012;109(24):9523-9528.

49. Benjamini Y, Drai D, Elmer G, Kafkafi N, Golani I. Controlling the false discovery rate in behavior genetics research. Behav Brain Res. 2001;125(1-2):279-284. 